\title{
Reflectance response of tapered optical fiber coated with graphene oxide nanostructured thin film for aqueous ethanol sensing
}

\begin{abstract}
In this work, optical sensing performance of tapered multimode fiber tip coated with graphene oxide (GO) nanostructured thin film towards aqueous ethanol with different concentrations is investigated. The tapering process of the optical fiber is done by a glass processing machine. The multimode optical fiber tip is dip-coated with GO and annealed at $70{ }^{\circ} \mathrm{C}$ to enhance the binding of the nanomaterials to the silica fiber. FESEM, Raman microscopy and XRD analyses are performed to micro-characterize the GO thin films. The morphology of the GO is observed to be in sheets forms. The reflectance response of the GO coated fiber tip is compared with the uncoated tip. The measurements are taken using a spectrophotometer in the optical wavelength range of $55010720 \mathrm{~nm}$. The reflectance response of the GO coated fiber tip reduced proportionally, upon exposure to ethanol with concentration range of $5 \mathrm{i} 80 \%$. The dynamic response of the developed sensor showed strong reversibility and repeatability when it is exposed to ethanol with concentrations of $5 \%, 20 \%$ and $40 \%$ in distilled water. At room temperature, the sensor shows fast response and recovery as low as 19 and $25 \mathrm{~s}$, respectively.
\end{abstract}

Keyword: Graphene oxide; Ethanol; Optical sensing; Reflectance; Optical fiber sensor; Dipcoating 